\title{
Responsible Research and Innovation
}

\author{
Critical reflection into the potential social consequences of ICT
}

\author{
Grace Eden, Marina Jirotka \\ Computer Science Department \\ University of Oxford \\ Oxford, UK \\ \{grace.eden, marina.jirotka\}@cs.ox.ac.uk
}

\author{
Bernd Stahl \\ Centre for Computing and Social Responsibility \\ De Montfort University \\ Leicester, UK \\ bstahl@dmu.ac.uk
}

\begin{abstract}
The potential social consequences that may arise as a result of the development and widespread use of ICT are of increasing interest to the general public, policymakers and researchers. Prominent examples include transformations of our concept of privacy when using social networking and other websites, ownership and control of personal data, and the ways crowd-sourced information transform how events are coordinated and how they unfold in real-time. Although there is broad acceptance that questions of professional responsibility are relevant to ICT it is often unclear how this could be achieved or how responsibilities should be defined and managed when considering the potential social consequences of ICT. 'Responsible Research and Innovation' (RRI) has emerged in Europe proposing approaches for researchers to identify and consider the potential social consequences and impact of their research outputs within the entire research and innovation lifecycle. First, it asks researchers to take on a practice of critical reflection considering the potential societal impacts of their research outputs, and second to include the general public in a dialogue around the development of research goals and strategy. We discuss findings from interviews conducted with a broad range of stakeholders regarding challenges to identifying, debating and resolving social and ethical concerns associated with ICT research and practice. We also consider how we might extend existing or develop new approaches that facilitate both critical reflection and wider participation within the entire research and innovation lifecycle.
\end{abstract}

Keywords-Professional responsibility; Social factors; Public engagement; Requirements Engineering.

\section{INTRODUCTION}

Many ICT practitioners and researchers are familiar with the concept of professional responsibility especially when they encounter 'codes of practice' either within the workplace or through membership in professional organisations such as the IEEE [1]. Members commit to conform to these codes and to apply the principles contained within them in their daily practices. The Responsible Research and Innovation (RRI) initiative is not concerned primarily with the development of yet another code of practice. Rather, it seeks to facilitate a more reflective and inclusive research and innovation lifecycle process from fundamental research through to application design. Within each phase of the innovation process there may be particular concerns. For instance, decisions made at the vision and strategy phase will steer the direction of research so that it might reach the long-term goals described. Following on from this, research outcomes will, in large part, determine the types of products and services developed by industry. In turn, these would then be made available for use by the general public. For each link in the chain there may be certain responsibilities associated with activities that occur within them, particularly in relation to how decisions taken might impact upon society. A recent example of how the innovation lifecycle might impact upon society can be found in the current debate around Google Glass where campaigners are warning that its use may infringe upon basic human rights (e.g privacy) [2]. RRI aims to address this entire process so that the potential impacts of novel technologies might be discussed early on in the innovation lifecycle by as wide a swath of stakeholders as possible. One of the first definitions of RRI is offered by [3] who suggests that it can be understood as:

A transparent, interactive process by which societal actors and innovators become mutually responsive to each other with a view to the (ethical) acceptability, sustainability and societal

desirability of the innovation process and its marketable products (in order to allow a proper embedding of scientific and technological advances in our society).

In other words, the RRI is meant to address the gap in time between the initial phases of research strategy formulation to the point at which individuals and organisations regularly use products or systems based upon research outputs. Other definitions do exist $[4,5]$ however the key component of each is that they all express a need to develop greater democratic accountability within the innovation lifecycle. In this way, stakeholders would be obliged to justify the reasons for a particular research strategy, project, outcome, product or service by identifying and discussing both the potential negative as well as positive societal consequences. Overall, RRI aims to improve upon existing processes of research governance with a view to ensuring the acceptability and even the desirability of research outcomes.

The argument put forward for the implementation of RRI by the European Commission Horizon 2020 framework (the next framework for funding research in the EU), is that future public concerns, not imagined by policymakers or researchers, could thwart either the further development or the up-take and use of research innovation. This, it might be argued, resonates with a market-based justification for RRI - by increasing the 
connection between science, technology and society the acceptance and acceptability of novel technologies could be increased. From within the range of RRI perspectives, whether its origins be moral (action based upon the quality of virtue), legal (action based upon avoidance of liability) or economic (action based upon markets), the two key attributes of RRI remain the same - to take into account the concerns and expectations of a broader set of stakeholders including the general public through the facilitation of public participation in research strategy and the equipping of a more reflective research culture.

RRI is being considered as a new criterion within the EU grant submissions process in Horizon 2020, where it may become a requirement for researchers in ICT, and science and technology more broadly, to investigate the potential social impacts of proposed research. This presents a new research challenge for ICT researchers who may be submitting proposals for research that would develop systems in surveillance, robotics, decision-support, social media and indeed all areas of ICT research.

In this context we have conducted investigations into how ICT researchers conceptualise social and professional responsibility. We have taken this orientation first rather than a focus on a method for implementing RRI because of the very novelty of RRI where there have been thoughtful, yet tentative, attempts at creating a systematic approach to identifying, discussing and resolving issues around the potential positive and negative social consequences of research. Within areas of the ICT landscape such as HCI, techniques have been developed under the heading of value-sensitive design [6]. However, these are commonly implemented within the software development process itself. RRI approaches, on the other hand, take a much broader view of the entire research and innovation lifecycle where activities are framed as being upstream (proposal writing, directed funding calls), midstream (activities that occur within a project - research process) and downstream (when the outcomes of research are commercialized into marketable products) [7]. For our research, in order to begin to develop a technique tailored for the ICT research community we first wanted to gain an understanding of current issues, challenges and attitudes. With this broader understanding our goal is to then co-develop an RRI technique or framework with the UK ICT community.

\section{RESPONSIBLE RESEARCH AND INNOVATION}

Information systems (IS) engineering technologies are increasingly becoming embedded into our work and home lives creating a complex interdependence between people and technology as individuals and groups communicate, collaborate and exchange knowledge through a variety of Information and Communication Technology (ICT) systems. We follow Paul's [8] suggestions to view information systems as information technology in use where ICTs are seen as the tools and IS the business processes that make use of ICT technologies. In this regard, information technology or, as we prefer, information and communication technology (ICT) may be seen as the technical aspect of the socio-technical systems that are used in society and organizations. Research and innovation thus provide the basis for the eventual use of IS. At the same time
IS are not determined by ICT but are open to negotiation and flexible interpretations.

Traditionally ICT research, design, and applications have been associated with the development of tools that possess discreet and transparent functionality meant to support specific tasks. However, today their 'diversity, scope, and complexity' has extended beyond the support of tasks and interaction to becoming situated within the very fabric of our daily lives [9]. Rather than being merely tools, the technologies we design are arguably transforming and augmenting the world around us, where computer-generated information, objects and infrastructures 'coexist in the same space as the real world' [10].

Today, ICT researchers are being placed into potentially precarious situations because they are increasingly being asked by funding bodies and public policymakers to become accountable for how their own research and development projects may impact upon the long-term shaping and transformation of society. The research challenges associated with identifying and understanding the potential broader societal impacts of technology have been discussed by computer scientists, social scientists and philosophers [11, 12, 13] through the avenues of Computer Ethics to the Social Construction of Technology: RRI has been influenced by both.

The field of computer ethics has developed in parallel to computing itself where the potential of computing technologies to raise ethical issues was visible from the start. Like computing, computer ethics, arose from, and retains references to, a number of different disciplines including computing science, engineering, philosophy and social studies of technology. From this disciplinary diversity one can find a broad range of theories, topics and research questions reflected. [14] argue that research in computer ethics is composed of an interest in the "ethical issues of privacy, security, computer crime, intellectual property, free expression, and equity and access, and issues of responsibility and professional ethics." Computer ethics has undertaken much conceptual work defining the ethical issues in ICT and linking it to philosophical ethics. There has also been a significant amount of empirical work exploring moral preferences and dilemmas $[15,16,17]$.

In the field of computer ethics there has been a longstanding debate about whether the activities and products of computing raise ethical issues that differ from general ethical concerns, that then warrant a new discipline of computer ethics [13]. This debate is still ongoing, and it provides us with a number of pointers to certain unique characteristics of ICT that might set it apart. For example, ICT may be characterized as particularly "malleable". This means that ICTs may be designed to conduct almost any activity that involves logical operations, input and output and thus, can be used in a wide variety of application areas. Whilst all technologies may be used in any number of different ways, the possible uses and misuses of ICTs are particularly difficult to predict due to this malleability.

A further distinguishing feature typical of ICT is what Johnson [18] calls "the problem of the many hands". This refers to organizational and institutional reliance on a division 
of labour where most activities are split up between numerous different individuals. This makes drawing a clear line between individual actions and eventual consequences difficult. It is further exacerbated as ICTs are increasingly interlinked and highly complex, making attribution of discrete features and functionality within an IS to individual researchers, developers or strategists often conceptually and empirically impossible.

Finally, the notion of "convergence" [19] refers to the increasingly pervasive nature of technologies where in the age of the internet, web 2.0 and pervasive computing, demarcating clear boundaries between systems, features and functionality becomes increasingly problematic. This includes most cuttingedge ICT-related research such as nanotechnology, biotechnology, and cognitive technologies. It is the potential interlink of these that might create a situation where the ability to freely choose the technologies one might adopt is curtailed. Convergence increases technological complexity, speeds up the pace of development through its combinatorial power, and also renders detailed accounts of their effects on social and ethical aspects difficult.

Computer ethics is a well-established field of study with streams of research in a variety of topics and an organisational infrastructure that includes conferences, journals, and research institutes. It remains in close contact with its reference disciplines but has also developed something of a disciplinary identity. However, despite its track record, several shortcomings with its discourse have been identified [20, 21]. Key among these is the question of practical relevance. Computer ethics may provide scholars with a wealth of theoretical insights into possible moral problems, ethical reflection, as well as meta-ethical positions with which to frame them. It draws upon a detailed understanding of the practicalities of ethics in ICT; however, it has had little impact on how these issues are discussed within society generally, or how relevant policies are developed and implemented. This lack of practical relevance may explain the emerging transition from computer ethics to responsible research and innovation in ICT. RRI is more specifically geared towards policy development and the establishment of governance mechanisms, drawing upon ethical analyses from different fields, but it goes beyond such analysis and establishes practically relevant activities.

The general conceptualisation of RRI first appeared in 2003 with the passing of the US ' 21 st Century Nanotechnology Research and Development Act' [22]. After which, the European Commission soon followed in 2004 with its communication 'Towards a European strategy for nanotechnology' [23]. RRI thus began with a clear aim to identify and address uncertainties and risks related to emerging research areas beginning with Nanotechnology [24] and moving into the environmental and health sciences including Geoengineering [25] and Synthetic Biology [26]. The scope of RRI has since expanded to include Computer Science, Robotics, Informatics and ICT more generally [27, 28].

\section{A. Framework for Responsible Research and Innovation}

In this paper we are interested in understanding how methods and techniques in RE might contribute to developing an appreciation of the wider contexts in which research and development outcomes are eventually embedded. This paper will also suggest that we may wish to draw on recent developments in RRI to extend or develop new approaches in $\mathrm{RE}$ to consider values in design, computer ethics, professional responsibility and the social consequences of ICT. We consider what such a synthesis might look like: where RE could inform the emerging RRI initiative, and also, how RE might be shaped in the light of the broad objectives of RRI.

As part of this research we are conducting three key activities: a landscape study (completed in 2012), the development of a community-driven website, and facilitation of group discussions with ICT researchers to begin to develop a RRI framework that could be embedded within the ICT research and innovation lifecycle. To date, there have been no comprehensive studies undertaken to gain an understanding of ICT researchers' perceptions of how they critically reflect upon their research outcomes or how they may or may not engage with the wider public. In 2012, we interviewed leading computer scientists, postdoctoral, researchers and $\mathrm{PhD}$ students in the UK. The interview or 'landscape study' provides the first extensive summary of current positions regarding the boundaries of professional responsibility as it relates to the identification of potential long-term societal consequences of ICTs. Our project will provide an important baseline giving us an opportunity to describe, understand and triangulate ICT researchers' issues and concerns across a variety of computer science domains such as; databases, human-computer interaction, mobile computing, artificial intelligence, and visualization, to name a few ${ }^{1}$.

This paper will discuss the preliminary findings of the landscape study where we interviewed computer science researchers working in a variety of domains. It will also make specific recommendations for how the ICT research community might begin to both develop and incorporate an RRI framework into everyday practice. The aim of our research is to identify emerging research challenges within the context of RRI and to discuss how we might address these.

\section{METHODOLOGY}

Our study has focused on two areas; first, 'upstream activities' that include top-down initiatives that shape strategic direction and research funding policy, and second, 'midstream activities' where R\&D and application design activities take place on the ground in actual projects. By developing an understanding of the landscape from these two perspectives we can begin to construct a picture of the current research and innovation cycle and ask how critical reflection regarding the potential social impacts of ICT currently takes place. A longterm goal of our project is to provide a set of recommendations and good practice that could be adopted by ICT researchers and application designers.

For the study we conducted semi-structured interviews with ICT researchers - those who seek funding, and with portfolio managers - those who manage research council funding calls, within the Engineering and Physical Sciences Research

1 Framework for Responsible Research \& Innovation in ICT, http://responsible-innovation.org.uk/frriict/ 
Council (EPSRC) ICT portfolio [29]. The goal of the interviews is twofold; in the case of researchers we are interested in finding out what their current perceptions are of their professional responsibility when considering the ethical and social implications of their research. We were also interested to find out the ways in which issues around professional and social responsibility are identified, discussed and resolved within research projects as well as their overall influence on research interests. In the case of portfolio managers we are interested in the processes and procedures that motivate the identification and direction of long-term funding strategy, which, in turn influence the development of themed funding calls. We also included corporations, professional bodies, and charities in the interviews in order to understand how their concerns might impact upon RRI.

We interviewed a range of ICT researchers with differing degrees of experience, from professors to doctoral students. In all, 67 interviews were conducted with an aim to discuss these issues with people involved in nearly every stage of the research, development and innovation lifecycle. This includes those working in research funding agencies, a range of people with different levels of experience in research, and potential user organizations (including charities, professional organisations, and industry). In total, we interviewed fortyeight UK academic computer science and related researchers; eleven funding body members; two NGO representatives; three corporate representatives, and three professional organization representatives.

We chose to conduct a breadth of interviews across as many computer science disciplines as possible to provide a broad overview of issues and concerns, rather than depth which would have provided insights from only two to four research areas. Additionally, conducting a breadth of interviews across ICT domains would ensure maximum outreach for both raising awareness of RRI and to begin the reflection process regarding current procedures and research practice. Interviews were audio recorded and ran between 60 to 90 minutes. The audio was transcribed and analysed using qualitative methods and informed by thematic analysis [30] where themes were identified and coded across interviews. The focus of analysis was the identification of reoccurring themes associated with concepts and activities that appear in interviewee transcripts in the form of opinions, reflections, conceptualisations and experiences. These convey the ways in which social and ethical challenges related to research practice are identified, debated and resolved. Although our study is wide ranging, for this paper we focus on three areas that might pose novel challenges and social consequences: human-robot-interaction, computer vision and ubiquitous computing. In what follows we discuss findings from our study that examine participants' perceptions of professional responsibility when considering potential longer-term social impacts. After which, we discuss the potential implications of our findings for ICT research and practice.

\section{THE LANDSCAPE STUDY}

\section{A. Contributing to the public good}

Both researchers and funders agree that ICT research contributes to the public good as one funding council portfolio manager notes:

That's our main driver. We have these themes set-up with perhaps specific funding calls, which would encourage people

to move towards them. ... Yea, it may not be as explicit as

saying at the level of a particular call. But the reason for

having the programmes and the strategies of funding would have been driven by: these are here for public good (Funder in ICT - 01).

For both ICT researchers and funders, contributions to the public good are determined by the description of 'impact' written into proposals. Descriptions of impact are meant to identify the potential beneficiaries of research (e.g. industry, government, charities), how they might benefit, and how a research project might engage with these stakeholders. However, researchers are not asked to consider broader, longterm societal risks, uncertainties and consequences that may be negative. Rather they are asked only to consider positive impacts, and how other researchers (in both academia and industry) might use their outputs when the research is completed.

Even though funders and researchers agree that research is funded for the purposes of contributing to the public good, both expressed a general distrust of involving the general public in any decision-making processes related to determining funding strategies. As one of the funders suggested:

It wouldn't be appropriate to put [the general public] into peer review. The idea that someone in the general public could know whether something in [portfolio research area] is going to transfer into their lives is ridiculous because I barely understand [it] sometimes. ... So it would be more appropriate for them to be involved in the higher levels ... When you're talking about high-level strategies; then there probably is a role for the general public to play (Funder in ICT - 03).

This view highlights the difficulties of involving the public in decisions concerning research strategy and impact. However, it may also not resonate with the experiences of some researchers in HCI, RE and other areas who work closely with user communities and the general public through prototyping and participatory design activities.

Neither, we the researchers or the user community can work in a vacuum. It's a case of saying 'here are some ideas about what one might do with the digital technology available and with what we think we can do with it. Here are some ideas, do you think they're good ideas. In the same one day workshop users are saying 'this is what we need', 'here there's a gap', 'here there's a lack'. And it's a case of marrying these two through the usual devices of workshops, stick-its, white boards. Coming together on workable research that has both an outcome that's of value in society and economy, and of 
course provides us with academic research of high quality

(Director, research unit -01).

Here we see three levels of possible broader stakeholder engagement: first, through the development of high-level strategy within the funding council; second, greater reflective practice in the peer review process; and third, at a project level using participatory design devices such as facilitated workshops.

One suggestion for linking RE with RRI might be through the ICT community's tradition in the use of HCI and RE methods for user engagement and general public participation within the software development process. Participatory approaches such as workshops and idea generation activities could serve as a model for what some may consider more fundamental computer science questions when developing research strategy, conducting peer review or engaging in participatory design. Take for example, how we might consider broader questions around social responsibility in fundamental research such as automating human reasoning in emergency response systems and applications? Automating reasoning is framed as a "scientific curiosity" as one of our interviewees described it. However, such research is funded to address societal needs, e.g. better coordinated and successful responses to emergency situations. Yet we may find situations where systems are required to make 'moral decisions' independently of humans related to logistics management, triage and delivery of care.

When discussing fundamental research questions, rather than engaging participants with prototypes, we might adopt a more conceptual approach, for example, motivating participants to think about potential outcomes. Take an emergency response scenario, what if automatic scene interpretation and object recognition from video footage generate representations of a scene that are incomplete or in some way misinterpreted by a system, or where that data is used by a system to make decisions in emergency situations? In this case, the motivations, intentions and purposes of such research could be more closely scrutinized to reveal not just the positive outcomes but also potential challenges of adopting this technology.

Here we see how scenario analysis, risk assessment and technology impact assessment [31] might be integrated into RE practice providing stakeholders an opportunity to identify, discuss and potentially resolve conceptually complex questions. We suggest that RE approaches could potentially be extended to facilitate discussions about the conceptual underpinnings of fundamental research by shifting the focus when using tools like scenario development and risk assessment.

\section{B. We are already responsible}

Many ICT researchers we interviewed expressed a belief that they are intrinsically ethical as this researcher notes:

I don't think I've come across an unethical scientist. ... if a problem occurs it's may be that they just haven 't considered the true implications of where there could be problems down the line. I think it's more that it hasn't occurred to them rather than they've considered it and decided to go with it for the hell of it anyway (Doctoral student, artificial intelligence).

Many times, researchers are assumed to be doing the most ethical types of work, adhering to traditional notions of professional responsibility such as experimental reproducibility, laboratory health and safety, data protection and obtaining informed consent. However, granting the benefit of the doubt to researchers that they are somehow inherently ethical could present risks in itself. One RRI approach, SocioTechnical Integration Research (STIR) [32] uses an intervention protocol (opportunity, considerations, alternatives, and outcome) to prompt researchers to think beyond the immediate technical procedures of a project. The purpose of STIR is to embed reflective mechanisms into day-to-day research work as it occurs in a setting. In this way, its aim is to facilitate a recalibration or modulation of a research path, if necessary, after taking into account unforeseen risks and uncertainties as they arise within the duration of a project.

In addition to assumptions that researchers are essentially already professionally responsible, another researcher expressed concerns about how RRI could have a negative effect on national prominence on the world stage within his discipline.

If the full context were present in the project and for some reason it was deemed unethical research within that context, then I'm sure that other people in the world would continue to be doing that kind of work (Professor, cybersecurity).

Competing on the global stage with colleagues is not only consequential to researchers, but it may also be critical, in this case, to national security. Here, funding bodies play a key role when they take into consideration the types of research to fund; they assess research activities on a global level, and determine national strength areas through metrics and consultation with research communities. Using these parameters as a guide, research challenges are identified and funding allocated. So in this sense, it might be argued that funding bodies could be equally, if not more, responsible for steering the direction of research as one researcher notes:

Research funded by government is trying to extend knowledge and application into areas that society want to see extended. It's not for academics to pursue whatever interests they want, they need to be able to relate that to why they are delivering

something that society actually wants to see (Director, research unit - 02).

In this way, we see how funding councils themselves are also responsible for the outputs of research as they steer the direction of research through themed funding calls. In addition, the peer review process has a responsibility to assess the 'impact' of research proposals using criteria such as: contribution to foundational research goals, developing proofof-concept systems in partnership with industry, public engagement and dissemination. It may be, given the nature of the exercise that researchers are encouraged to think about impact from an optimistic point of view rather than considering the potential long-term social consequences. RRI aims to 
manage novel innovations that are deemed to have highly uncertain future impacts and where no precedent exists and so, it is argued that because of this criterion, professional responsibility cannot be presumed.

Even though researchers consider themselves responsible, those we interviewed also said they $d o$ confront ethical dilemmas in their day-to-day projects. For instance, some expressed concern about creating a surveillance society, others discussed their unease about which types of data might be acceptable to divulge to users (e.g. physiological monitoring in gaming applications), some spoke of systems that take away people's jobs replacing humans with machines, those in robotics talked of debates in their community about whether patients using assistive healthcare robots should have the ability to turn them off, others were clear about the types of military research acceptable to them (e.g. systems for threat detection or doing harm), concerns over animal welfare with the use of insects in robotics/AI research, and the limits of automated reasoning (e.g. restricting human creativity and decision-making). In addition to these challenges, ICTs have an immense role to play in changing behaviour as one researcher stated:

There are moral challenges because you're using software in order to change behavior of the community your aiming your software at. And if you're changing behavior then you have to understand what the overall impact of the changes to behavior will be. So if you are trying to encourage a behavioural change through the software there better be and an understanding of what that behavioural change will mean. And also, what kinds of changes in behavior will be required for that software to be deployable and usable (Professor, distributed systems - 01).

Some researchers frame these challenges as moral dilemmas others, as practical dilemmas. Whether moral, or practical, many of the researchers we interviewed were initially resistant to the idea of thinking through the potential challenging effects of their research. Towards the end of many interviews however, some acknowledged that they simply have never been asked to consider these kinds of questions.

Although RRI shares many of the same techniques as RE such as workshops, focus groups and scenarios the orientation is on developing improved research governance mechanisms. However, within RE and HCI the orientation is towards developing a detailed and sequential understanding of a problem space. To date, RRI research has been conducted by social scientists and philosophers however the RE orientation towards problem solving is engineering-based. We suggest that RRI could be informed by RE in the examination of the motivations, intentions, and purposes of research especially when attempting to identify potential misuse cases. So rather than framing the discourse through the lens of 'responsibility' it could be framed as a 'problem space'. In this way RRI might be seen as a resource for innovation rather than a constraint.

For example, in 'privacy by design', misuse scenarios are used to steer the direction of the research and innovation lifecycle to ensure a system complies with data protection. Researchers develop misuse scenarios to identify risks after which, they develop solutions (e.g. data minimisation) that address those risks. This reframing from 'responsibility' to 'problem space' may allow ICT researchers to engage more readily in activities that not only encourage reflection but also facilitate the creation of candidate solutions during the first stages of innovation.

\section{Societal risks must be obvious}

On the whole, ICT researchers take a very strong stance around the notion of the 'obviousness of risk' in relation to the prediction of potential future outcomes of technology. This appears to produce a tension with the RRI aim to manage novel innovations that are deemed to have highly uncertain future impacts and where no precedent exists because these may not be readily obvious. In our interviews, researchers express concerns that it may not be appropriate within the context of computer science research to try to predict future impacts. In particular researchers cite two unknown factors in prediction; for fundamental research - societal consequences are perceived as being identifiable only within their contexts of use. And for application-oriented research - the possibilities of industry and user adaptation may change the trajectory of a technology in unforeseen ways. One researcher notes:

The research that we do, doesn't inherently tell you how to use this research. So, we built a robot that can throw balls six meters compared to one meter from previous versions. So, there is no kind of moral value associated with that, it's more of a performance. We're focused on the performance. How you use that performance is not our part I think (Postdoctoral researcher, robotics).

This researcher considers his work to be fundamental research and we find that many who self-identify as fundamental researchers also express a similar view; the implication here seems to be that the technology itself appears to be benign or agnostic, without any social values present in its outputs. In other words, social values manifest when the technologies are put to use within specific contexts. The same researcher goes on to say:

My work focuses on ways in which we can control impedance. So, part of our project is working out how to control robots and part is trying to understand how humans control their impedance and transfer those ideas on to robots (Postdoctoral researcher, robotics).

The fundamental research question and technical challenge is 'how can robots move more efficiently through space'. However, achievements in increased robot mobility and range of movement do have implications for human-robot interaction in a range of contexts such as home, work, military, and in public settings. RRI would contend that rather than assume novel technologies are value-free; researchers should be encouraged to anticipate future use through reflection within the research community and deliberation with a wide group of stakeholders. One RRI approach to this is called 'stage-gating' [25], a project management technique for managing investment risk. Using this technique, decision points are introduced into a research project lifecycle where at set intervals stakeholders are asked to reflect upon the purposes, motivations and products of 
research. These are then assessed by an independent stage-gate panel and recommendations fed back to funders regarding how, and if, a research trajectory should be redefined or changed. This is a more formalized approach to STIR, which is conducted informally by the researchers in a setting, whereas the stage-gating technique brings in external assessors who report back to the funders.

Such RRI approaches may be useful in research areas where solutions are being developed for difficult technical challenges; many times at the expense of considering what possible futures research outputs might facilitate. In these cases, where the technical challenges are at the forefront, there is a tendency for many researchers and funders to believe that fundamental research is somehow independent or very far removed from society. We see this occur in two circumstances. First, when research outputs contribute to the development of 'enabling' or 'generic' technologies that could be adopted across a wide variety of domains and contexts (e.g. healthcare, military, industrial or domestic settings). And second, when researchers feel that their projects do not engage directly with potential user communities.

In contrast, for those who conduct application-oriented research, the potential social consequences of their outputs might be more obvious to them as this researcher working in assistive human-robot interaction says:

The question is if you had a robot in the home, with an arm, with a manipulator that can pick up these objects and then

bring it to the person, how do people want the robot to behave. When should the robot approach people, how should the robot interact with the person, how should the robot transport these objects, how should the robot hand over these objects to a person. These are all things we explore in certain conditions ... The question is, from the person's point of view, would they accept that. These are issues that are not only technological problems but they depend on what people would prefer. What are people's perceptions of these machines, what are their expectations, what are their prejudices? Are they concerned with the movement or the size ... It has to do with people's perceptions of robots, if they accept these machines, and that informs us as designers. (Professor, human-robot interaction).

Although the technical challenges of designing robots are considerable, focusing solely on the optimisation of 'performance' is not sufficient as the quote above suggests. In addition to performance, the ways in which robots interact with people under different conditions may determine how successful their integration into various settings might be. This wider consideration takes into account not only the technical, but also the sociocultural and interactional challenges.

Even with this sensitization towards sociocultural concerns, application-oriented researchers, similar to fundamental researchers, stated that potential risks and uncertainties should be obvious before any decisions about whether or not to pursue a research path is made. As the same researcher states:

Any research can always have negative consequences. We take a lot of precautions in what we are doing but you can never fully predict whether there might be negative consequences or not. It would be completely unrealistic and dishonest if I said; 'oh, there will never be any negative consequences of my research'. You can't say that. Who knows how someone else might use our research. You can never predict (Professor, human-robot interaction).

For many researchers whom we interviewed, the most obvious issues related to professional responsibility are associated with traditional understandings such as experimental reproducibility, laboratory health and safety, data protection (e.g. security, confidentiality and privacy of personal data), and obtaining informed consent. Outside of these three broad areas: the implementation of correct procedures, data storage and use, and human subjects considerations, many researchers and funders we interviewed struggle to identify potential negative consequences of ICT research.

Interestingly, ICT researchers have been investigating the use of scenarios to identify potential challenges with future implementation of technologies [33, 34], and others in RE have suggested the use of foresight techniques [35]. Even so, we find that many researchers may be reluctant to engage in critical reflection where they, at times implied, and at other times explicitly stated that if such descriptions were made public and written into proposals it might adversely effect their successes at gaining funding. An alternative to creating such a situation may be to encourage open reflection and public debate without necessarily including those descriptions into proposals. This may relieve researchers' anxieties about risks to receiving funding but it might not resonate with some advocates of the RRI initiative who promote the creation of formal rules around research governance.

A compromise solution could be the use of Constructive Technology Assessment (CTA) [36], which examines research strategy in relation to institutional dynamics, rather than individual actions. Giving researchers the space to be 'one step removed' may soothe their inhibitions allowing them to speak more freely about the potential effects of ICT research on society. Such an approach may be especially useful beyond the design of hardware and software, in areas such as device manufacturing where concerns are expressed regarding the prediction of environmental impacts. However, one researcher suggested that because he is not directly involved in the manufacturing process those issues are of no direct concern to his research.

I don't specify how things get made. I specify what I want on the chip and then the manufacturers are responsible for putting it there by whatever means they use (Professor, microelectronics).

Here the researcher frames the question of responsibility solely from a personal perspective. However, by using techniques such as CTA, the researcher would be allowed to reflect upon the institutional dynamics associated with chip manufacturing. This could enable discussion around concerns with the use of rare earth minerals and the release of toxic materials in the production processes with a wide group of stakeholders. Interestingly, one researcher working in mobile 
device and infrastructure design told us that a research challenge in his domain is the sourcing of alternative materials and reduction of the $\mathrm{CO}_{2}$ footprint through more efficient energy consumption.

The next thing we are going to get into is the precious metals and the limited resources we've got in terms of some of the compounds and chemicals that we've got on the planet. There is not a bottom-less pit. The biggest problem today is the great throwaway society. Everyone has to have the latest gizmo and product (Professor, mobile computing).

Here we see that these two researchers differ in what they feel may be their professional responsibility. In the first instance, the obviousness of risk and uncertainty related to energy use, mining of precious metals, and toxic by-products was less of a concern than to the second. Today, the use of water, fossil fuels, and chemical process is of increasing concern to the ICT community [37] and perhaps if a forum of ICT researchers were to be brought together using the CTA approach, peers within the community would feel less threatened that they, personally, may be criticized for not being professionally responsible. Rather, the focus would be placed upon institutional dynamics so that solutions could be discussed for how best to change them.

We found in our interviews that when researchers and funders discuss potential social consequences of research they habitually focus on the positive impacts to people, society and the economy, possibly at the risk of not addressing much more subtle, but equally important consequences. There is reluctance towards trying to predict potential impacts or the use foresight methodologies to speculate about possible future social consequences. This may be because science is based upon empirical evidence, whereas future scenarios are used to imagine possibilities. These are two very different approaches to problem solving (anticipatory vs. evidence-based). Recognizing these differences, it is important that all stakeholders rather than a single stakeholder formulates the questions used to develop scenarios.

Additionally, we argue that the RE community has much to contribute in the development of suitable approaches for all stakeholders to debate these issues (researchers, funders, industry, charities, and the general public). Taking into account the significant expertise already in place, the RE community has access to, and continues to build upon, a range of techniques for eliciting people's opinions, ideas, and approaches to conducting their work. Therefore, we suggest that RE is uniquely positioned to extend and modify existing techniques to be used in this ICT context.

\section{Responsibility in fundamental and applied research}

As stated above, a noteworthy distinction has consistently appeared across both funder and researcher interviews that may promote current perceptions of professional responsibility. For many researchers there is a correlation between the location of their research within the spectrum of 'fundamental' to 'applied' and how, or even if, they should be concerned with the potential societal consequences of research outputs. Beliefs about where within this spectrum their research lies are used to judge whether or not long-term social responsibilities need to be considered. This distinction has implications for ICT research practice because it directs the focus of professional responsibility entirely on to those who design applications for specific contexts of use. As a researcher working in fundamental research in natural language processing commented:

To be perfectly honest, it's not something I think about. The projects I'm involved in, with maybe one exception, are basic research and will not have immediate impact on society. I know the EPSRC's all about impact and various things you can do to enhance impact. But, to be perfectly honest, that is not something I really-is not a day-to-day consideration (Reader, natural language processing).

Although, 'impact' refers to the effects that funded research may have, many who describe their research as fundamental claim that there is a caveat to judging their outputs because there are no guarantees that it will even be successful, which, they say, is why it is called 'research'. Research is conceptualized as finding answers through a process of trial and error and viewed as somehow separate from the concerns of day-to-day life because its focus is on extending knowledge of what may or may not be possible rather than producing determinable outcomes.

Fundamental research includes activities where basic principles are identified, technological concepts formulated and experimental research conducted to validate those principles and concepts. Application-oriented research, on the other hand, focuses on the practical application of those principles and concepts. The distinction has been articulated as that between the prototype, developed in application-oriented research, which is a working system that could, quite soon, be iterated into a fully developed product and the demonstrator, developed in fundamental research, whose purpose is to show the utility of the science with its potential commercialisation many years in the future.

In contrast to the interviewee above, one researcher noted that:

The holy grail [is] how to adapt systems to fit with people's context ... [and to] make computing fit into the background like furniture (Reader, ubiqitous computing).

Comparing the two quotes we see that the researcher engaged in 'fundamental' research has little or no concern for the social consequences of his research outputs. Whereas, the 'applied' researcher has these concerns at the forefront where they are utilised to develop the most appropriate and usable technologies. This generalization regarding the differences in attitude between fundamental and applied researchers is based upon what the majority of application-oriented and fundamental ICT researchers said during the interviews.

Even with this distinction, most researchers we interviewed who work in computer science departments describe their projects as a mix between fundamental and applied research:

Anything in [computer] vision is applied; it's the underlying mathematics that's basic (Professor, machine vision). 
Within projects we find that fundamental research, which may focus on algorithm and infrastructure design, is conducted alongside software engineering and interface design in domains such as energy, cybersecurity, and remote healthcare for example. Importantly, many projects within computer science research could be viewed as falling between both ends of the spectrum.

We do look for applications as a vehicle for developing ideas rather than as an end in themselves. I try to identify a difficult problem and then think of a domain where I can feasibly tackle that problem (Professor, verification and correctness).

Even though this is the case, we find that many researchers, when asked about social responsibility, distanced themselves from any kind of obligation to critically reflect upon such questions giving a justification that their research was fundamental and so somehow outside the sphere of social accountability.

These are basically scientific questions that you're asking as opposed to 'will that lead to public good'. I think it's quite removed from that. When you start asking questions I don't think I think in terms of 'will this lead to anything that can be applied or tangible' at that stage. No, I don't ask that question (Professor, distributed systems - 02).

This sentiment has been voiced consistently in our interviews with fundamental researchers and may be related to the opinion that the outcomes of basic research are difficult to predict especially since it is unknown whether those outputs would be leveraged by industries in the development of new products or services. Some researchers also distance themselves from considering aspects of social responsibility by diminishing and downplaying the actual usefulness of their research outputs in both the near and long term.

\section{If our basic approach, constructing brain models one neuron at a time, if that's the right way to build an electronic analogue of the human brain, then it's fairly easy to predict where we'll end up with. That is: a computer that occupies a \\ large hall, consumes megawatts of power and would be completely non-portable and be a very long way from something you could stick on a mobile robot or connect it through sensors through a lab (Professor, many-core processing).}

This statement might be seen to diminish the potential societal effects of novel technologies to both placate apprehensions and to maintain some distance between research goals and social responsibility. Calvert [38] has shown that researchers use the distinction between fundamental and application-oriented research for several reasons such as, preserving professional status, maintaining autonomy and control, and preventing external evaluation and critique. However, when we asked researchers and funders they both acknowledged that even basic and blue skies research is justified precisely because of its potential to contribute to society, industry, and for providing economic benefit in the future.
Despite this, there remains a tension where researchers employ the fundamental-applied spectrum to measure their professional responsibility. When discussing actual projects it is used as a rough measure for a) whether research outputs might have either near or long-term uptake and use, and extrapolating from this, b) whether or not they should be responsible for considering potential negative social impacts. This leads to a series of often tacit presumptions for many of our interviewees about their professional responsibility and whether or not they should consider how their research may lead to more challenging consequences for society. This rationale is consistently used to determine if research outputs will be relevant in the creation of near and long term futures; the closer technologies are to being taken up by or embedded within society, the greater the societal impact.

This fundamental-applied dichotomy may be of greater consequence for those on the application-oriented side of ICT research because it suggests that questions of professional responsibility and societal impact might only be an HCI, Requirements Engineering and user acceptance problem for example, and not questions for fundamental researchers to consider. If this is the case, it presents many applicationoriented ICT researchers with a conundrum where they are asked to design applications based upon novel technologies after critical decisions have already been made about their social value.

\section{DISCUSSION AND FURTHER WORK}

The conceptualization and implementation of RRI presents many research challenges but also many opportunities for the ICT research community. We consider the practical implications of how RRI might influence the shaping of ICT research strategy and practice and also, how the ICT research community might shape RRI in the light of its broad objectives.

A key contribution the ICT community can make lies in its strong tradition in design through stakeholder participation. This could give RRI practical relevance especially through the $\mathrm{RE}$ orientation towards problem solving, which is engineeringbased. Rather than framing the discourse through the lens of 'responsibility' it could be framed as a 'problem space'. In this way, RRI might be seen as a resource for innovation rather than a constraint and could enable ICT researchers to engage more readily with potential negative consequences as a 'problem space'. This would allow them to not only reflect upon their research practice but also become empowered to change it through the creation of candidate solutions.

Additionally, RE might be influenced by RRI by, when necessary, potentially shifting its focus from detailed and sequential understandings of a system towards broader societal impacts when using scenario analysis, risk assessment and technology impact assessment. This might provide stakeholders an opportunity to identify, discuss and potentially resolve conceptually complex questions. We suggest that RE approaches could be extended to facilitate discussions about the conceptual underpinnings of fundamental research simply by shifting the focus when using existing RE tools. On the other hand, the RE and HCI communities have access to, and continue to build upon a range of techniques and methods for 
eliciting requirements, evaluating for usability and assessing values in design. Therefore it is uniquely positioned to further develop approaches and methods that could be used to consider issues in RRI.

Currently, various RRI frameworks are undergoing development [39, 40, 41] and are being designed mainly by researchers in philosophy (applied ethics) and public policy. Within ICT we suggest that the research community has an opportunity to contribute to the development of novel RRI methodologies so that research strategy decisions might be made not exclusively on the grounds of their technical or scientific attributes alone but to also consider potential social impacts. This would provide an opportunity for the ICT community to become directly involved with researchers asking similar questions in applied ethics, social science and public policy research.

As a first step in co-developing an ICT RRI Framework we propose that $\mathrm{RE}$ researchers might try using existing RRI techniques such as STIR, Stage-gating, and CTA. Often, the same techniques are used as in RE such as workshops, focus groups and scenario development. However, as the focus of formal RRI is on developing improved research governance mechanisms, the RE approach might be framed as a more practical approach used for problem-solving. Taking into account the significant expertise within the RE community for eliciting people's opinions, ideas, and approaches to conducting their work we argue that RE is uniquely positioned to extend and modify existing techniques that could be used within an ICT context.

Information systems research has always been an interdisciplinary endeavour, however, we maintain that a new kind of interdisciplinary practice be deliberated within our community. We suggest that the nature of the interdisciplinary collaboration itself be expanded beyond the requirements, user testing, and evaluation of computing systems. Rather, the ICT community could have a much more integral role to play when decisions are made about the very motivations, intentions, and purposes of computing research that are currently developed by policymakers and research councils.

This present research challenges, but we suggest that the ICT community, in particular RE and HCI, are the most appropriately positioned sub-disciplines of computer science to perhaps take a lead in facilitating the formulation of frameworks and guidelines. Such new approaches could assist members of research projects in developing mechanisms for critical reflection of research goals and strategies and ways to include wider stakeholder populations in the decision-making process. This is especially true since eventually, the outputs of fundamental computer science research will become the new challenges for application-oriented research and design and so it follows that RRI be developed in partnership with the ICT community alongside philosophers, social scientists and policymakers.

A further research challenge is that ICT researchers primarily consider meeting professional responsibilities through adhering to traditional notions of experimental reproducibility, laboratory health and safety, data protection and obtaining informed consent. However, these are related to research process, activities done within the scope of actual projects. Whereas, RRI aims to extend the notion of professional responsibility to include research products, including how ICTs might transform people and society. Currently, these questions are discussed downstream when products and services enter the marketplace. Many RRI advocates suggest that this may be too late in the innovation lifecycle. Referred to as the Collingridge dilemma [42] it creates a situation where the development of novel ICTs occurs within a double bind. The first is the 'information problem', where social consequences cannot be predicted reliably until ICTs are extensively developed and used. The second is the 'power problem' where changing technology becomes difficult once it has been embedded into society. We suggest that ICT researchers, who are members of actual research projects, can also contribute to debates about how research outputs may lead to unintended future consequences.

Generally we found that there is reluctance towards trying to predict potential impacts or the use foresight methodologies to speculate about possible future social consequences. This may be attributed to the different conceptual focus taken by ICT researchers and RRI advocates in the process of problem solving. RRI is anticipatory and uses future scenarios to imagine possibilities, whereas ICT is a science that uses empirical findings to make evidence-based decisions. Recognizing these differences, we suggest that the RE and HCI communities could begin to forge a synthesis where techniques and approaches in these domains could inform the emerging RRI initiative, and also, where RE and HCI might be shaped in the light of the broader objectives of RRI.

As a follow up to the landscape study we will be developing an RRI Framework for ICT. The framework will be informed through a series of focus groups and participatory sessions with the ICT research community to be conducted this year. The framework will contain two main guidelines: first, techniques for promoting reflection amongst researchers about project outputs and second, approaches to facilitate engagement with wider stakeholder groups in a dialogue about the societal consequences of research.

Additionally, we aim to identify good practice that may already be in place across the ICT community that could inform the Responsible Research and Innovation Framework. We will also develop an online resource, the Observatory for Responsible Research and Innovation which is being populated with case studies that discuss ethical and social dilemmas within specific contexts such as; the collection of shared data in social networking sites, new models for dynamic informed consent in online settings, considerations for long-term qualitative research in living labs, data aggregation and its implications for privacy, and best practice for conducting design activities with children [43].

In terms of ICT research policy, our research will also directly inform decision makers at the research councils. If the ICT portfolio introduces a RRI framework it may have policy implications as the funding councils might use its outputs to report back to the government justifications for the development of its research strategies and objectives. Whether 
or not a RRI framework is introduced will also depend very much upon the ICT research community.

The aim of RRI requires deep reflection about choices made regarding the direction of research investments, and accountability mechanisms to ensure that those managing and conducting research seriously consider potential societal impacts; that they are identified, discussed and addressed through the entire research and innovation lifecycle - from funding calls, to proposal writing, to peer review, to research outputs. RRI is a broad initiative that reaches beyond projectby-project concerns to the broader motivations and rationales that form government policy and influence funding provision. The intention of RRI is to ask the research community to reflect upon questions related to whether we proceed with a particular research agenda? If so, how do we proceed? And how should such decisions be made? [23]. The RRI focus is more on mitigating wider societal long-term risks and so favors incremental rather than radical innovation.

Additionally, the RRI initiative maintains that both our short-term (project-by-project) and long-term (grand challenges) research strategies, goals and outputs need to be continually reassessed and that fresh points of view need to be added to discussions and decision-making. Questions of the societal impact of ICT might be seen as an issue for policymakers and not for researchers to consider. If this is the case, it presents researchers with a conundrum where we are asked to design applications for technologies after critical decisions have already been made that have social and ethical consequences embedded within them. Finally, establishing the trajectory of the future on merely one account (e.g. looking at only the potential positive impacts of research) may not be in itself a responsible way to proceed. There are potential negative impacts to every imagined positive one. Because this is the case, diverse points of view can be complimentary to one another like photographs or videos of an event taken from various points. Each differing perspective provides us with new information that is not available from the other points of view.

\section{ACKNOWLEDGMENTS}

We would like to thank the FRRIICT advisory board, the core members of the project, and the wider network of ICT researchers who have participated in interviews and workshops. Additionally, we thank the Engineering and Physical Sciences Research Council (EPSRC) for funding the Framework for Responsible Research and Innovation project Grant No. EP/J000019/1.

\section{REFERENCES}

[1] IEEE Code of Ethics, http://www.ieee.org/about/corporate/governance/p7-8.html.

[2] BBC news, "Privacy 'impossible' with Google Glass warn campaigners," BBC news website, 2013, [http://www.bbc.co.uk/news/technology21937145, accessed 27 March 2013].

[3] R. Von Schomberg, "A vision of responsible innovation," In: R. Owen, M. Heintz and J Bessant (eds.) Responsible Innovation, London: Wiley., in press.

[4] R. Owen, P. Phil Macnaghten, and J. Stilgoe, "Responsible research and innovation: From science in society to science for society, with society," Science and Public Policy 39, pp. 751-760, 2012.
[5] H. Sutcliffe, "A report on Responsible Research and Innovation for the European Commission", 2011, [http://ec.europa.eu/research/sciencesociety/document_library/pdf_06/rri-report-hilary-sutcliffe_en.pdf, accessed 27 March 2013].

[6] B. Friedman,and P. H. Kahn, "Human values, ethics, and design,” In J. A. Jacko and A. Sears (eds.), The Human-Computer Interaction Handbook, Mahwah Erlbaum, pp. 1177-1201, 2003

[7] E. Fisher, R. L. Mahajan, and C. Mitcham, "Midstream Modulation of Technology: Governance From Within," Bulletin of Science, Technology \& Society, vol. 26,6, pp. 485-496, 2006.

[8] R. J. Paul, "Loose Change," European Journal of Information Systems, vol. 19, 4, pp. 379-381, 2010.

[9] A. Sellen, Y. Rogers, R. Harper, and T. Rodden, "Reflecting human values in the digital age," Communications of ACM, vol. 52, 3, pp. 5866, 2009.

[10] R. Azuma, Y. Baillot, R. Behringer, S. Feiner, S. Julier, and B. MacIntyre. "Recent Advanced in Augmented Reality," IEEE Computer Graphics and Applications, vol. 21, 6, pp. 34-47, 2001.

[11] N. Wiener, The Human Use of Human Beings. Da Capo Press, 1954.

[12] W. Bijker, Of Bicycles, Bakelites and Bulbs. MIT Press, 1995.

[13] J. Moor, "What is computer ethics?," Metaphilosophy, vol. 16, pp. 266275, 1985.

[14] P. Brey, and J.H. Soraker.. "Philosophy of Computing and Information Technology," In Philosophy of Technology and Engineering Sciences: 9, D.M. Gabbay, A.W.M. Meijers, J. Woods, and P. Thagard (eds). North Holland, pp. 1341-1408, 2009.

[15] D. Banerjee, T. P. Cronan, and T. W. Jones, "Modeling IT ethics: A study in situational ethics," MIS Quarterly, vol. 22, 1, pp. 31-60, 1998.

[16] T. T. Moores, and J. C.Chang, "Ethical decision making in software piracy: Initial development and test of a four-component model," MIS Quarterly, vol. 30, 1, pp. 167-180, 2006.

[17] T. Vartiainen, "Moral conflicts in project-based learning in ISD," Information Technology \& People, vol. 23, 3, pp. 265-280, 2010.

[18] D.G. Johnson, Computer Ethics, 3rd edition. Upper Saddle River, New Jersey: Prentice Hall, 2001.

[19] A. Grunwald, "Converging technologies: Visions, increased contingencies of the conditio humana, and search for orientation," Futures, vol 39, 4, pp. 380-392, 2007.

[20] T. W. Bynum, "Philosophy in the Information Age," Metaphilosophy, vol. 41, 3, pp. 420-442, 2010.

[21] S. Rogerson, "Ethics and ICT," In The Oxford Handbook of Management Information Systems: Critical Perspectives and New Directions, R. D. Galliers \& W. Currie Eds. Oxford: Oxford University Press, 2011, pp. 601-622.

[22] 21st Century Nanotechnology Research and Development Act. Public Law 108-153, 108th US Congress, http://thomas.loc.gov/cgibin/bdquery/z?d108:S189, 2003.

[23] European Commission. Towards a European strategy for nanotechnology,http://ec.europa.eu/nanotechnology/pdf/nano_com_en_n ew.pdf, 2004.

[24] K. Grieger, A. Baun, and R. Owen, "Redefining risk research needs for nanomaterials," Journal of Nanoparticle Research, vol. 12, 2, pp. 383392, 2010.

[25] P.M. Macnaghten, and R. Owen, "Good Governance for Geoengineering," Nature, vol. 479, p. 293, 2011.

[26] J.B., Tucker, and R.A. Zailnskas, "The Promises and Perils of Synthetic Biology," The New Atlantis, pp. 25-45, 2006.

[27] B.C. Stahl, G. Eden, and M. Jirotka, "Responsible research and innovation in Information and Communication Technology," In Responsible Innovation, R. Owen, J., Bessant, M. Heintz, Eds. London: Wiley, in press.

[28] J. Stilgoe, R. Owen, and P. Macnaghten, "An Outline Framework for Responsible Innovation: taking care of the future through collective stewardship of science and innovation in the present," EPSRC Technical Report, 2012. 
[29] Engineering and Physical Sciences Research Council (EPSRC) ICT, [http://www.epsrc.ac.uk/research/ourportfolio/themes/ict/Pages/ict.aspx, accessed 27 March 2013].

[30] G. Guest, K.M. MacQueen, and E. Namey, Applied thematic analysis. Thousand Oaks, CA: Sage Publications, 2012.

[31] T.A. Tran, and T. Dain, "A taxonomic review of methods and tools applied in technology assessment," Technological Forecasting \& Social Change, vol. 75, pp. 1396-1405, 2008.

[32] E. Fisher, "Ethnographic Invention: Probing the Capacity of Laboratory Decisions," Nanoethics, vol. 1, 2, pp. 155-165, 2007.

[33] C. Mancini, Y. Rogers, A.K. Bandara, T. Coe, L. Jedrzejczyk, A.N Joinson, B.A. Price, K. Thomas, and B. Nuseibeh, "ContraVision: Exploring Users' Reactions to Futuristic Technology,” ACM Conference on Human Factors in Computing Systems (CHI) 2010, pp. 153-162, 2010.

[34] W. Odom, J. Zimmerman, S. Davidoff, J. Forlizzi, A.K. Dey, and M.K Lee, "A Fieldwork of the Future with User Enactments," Designing Interactive Systems (DIS) 2012, pp. 338-347, 2012.

[35] J. Pimentel, J. Castro, H. Perrelli, E. Santos, and X. Franch, "Towards Anticipating Requirements Changes through Studies of the Future,' Research Challenges in Information Science (RCIS), 2011 Fifth International Conference, pp. 1-11, 2011.
[36] J. Schot, and A. Rip, "The Past and Future of Constructive Technology Assessment," Technological Forecasting and Social Change, vol. 54, pp.251-268, 1997.

[37] E.D. Williams, R.U. Ayres, and M. Heller, "The 1.7 Kilogram Microchip: Energy and Material Use in the Production of Semiconductor Devices,” Environ. Sci. Technol, vol. 36, pp. 5504-5510, 2002.

[38] J. Calvert, "What's special about basic research?," Science, Technology \& Human Values, vol. 31,2, pp. 199-220, 2006.

[39] P.A.E. Brey, "Anticipating ethical issues in emerging IT," Ethics and Information Technology, pp. 1-13, 2012

[40] J. Stilgoe, R. Owen, and P. Macnaghten, "An Outline Framework for Responsible Innovation: taking care of the future through collective stewardship of science and innovation in the present", EPSRC Technical Report, 2012.

[41] R. Von Schomberg, "Prospects for Technology Assessment in a framework of responsible research and innovation," In M. Dusseldorp, and R. Beecroft (eds). Technikfolgen abschätzen lehren: Bildungspotenziale transdisziplinärer Methoden,Wiesbaden: Springer Verlag, pp. 39-61, 2011.

[42] D. Collingridge, The Social Control of Technology. London: Francis Pinter Ltd, 1980.

[43] FRRIICT, online resource case studies, http://torrii.responsibleinnovation.org.uk/case-studies-1st-round. 